\title{
Understanding Digestive Ripening of Ligand-Stabilized, Charged Metal Nanoparticles
}

\author{
José A. Manzanares, ${ }^{*} \dagger \odot$ Pekka Peljo, ${ }^{\ddagger}$ and Hubert H. Girault ${ }^{\ddagger}$ \\ ${ }^{\dagger}$ Department of Thermodynamics, Faculty of Physics, University of Valencia, c/Dr. Moliner, 50, E-46100 Burjasot, Spain \\ ${ }^{\ddagger}$ Laboratoire d’Electrochimie Physique et Analytique, École Polytechnique Fédérale de Lausanne, EPFL Valais Wallis, Rue de \\ l'Industrie 17, Case Postale 440, CH-1951 Sion, Switzerland
}

\section{Supporting Information}

ABSTRACT: Most syntheses of thiolate-protected metal nanoparticles (NPs) include a thermochemical step in which the as-prepared, polydisperse NPs are transformed to a narrower size distribution in a poorly understood process known as digestive ripening (DR). Previous theoretical approaches considered either surface and electrostatic contributions or surface and ligand-binding contributions. We show that the three contributions are needed to obtain theoretical predictions in agreement with experimental

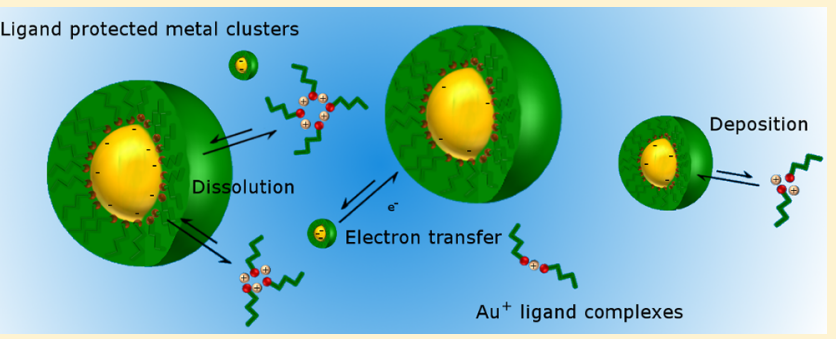
observations. Although statistical thermodynamics does not clarify mechanistic details, it certainly provides valuable insights on the DR process. Remarkably, a relatively simple theory with no fitting parameters satisfactorily explains the roles of the metal:ligand ratio, the NP charge, the relative permittivity of the solvent, the ripening temperature, the binding energy, and the ligand chain length.

\section{INTRODUCTION}

Ligand-stabilized metal nanoparticles (NPs) have become essential in many active areas of research. Although quite a number of chemical methods are currently available for their synthesis, only a few have an established position due to their facility to produce large quantities, not only with narrow size distributions but also with well-defined surface chemistry. ${ }^{1}$ Three well-known methods are (i) Brust-Schiffrin,, ${ }^{1,2}$ which uses an aqueous-organic two-phase system to reduce a gold salt in the presence of alkyl thiols, (ii) Turkevich, ${ }^{3}$ which involves boiling a gold salt with citrate, and (iii) solvated metal atom dispersion (SMAD), which involves the growth of clusters by co-condensation of metal and solvent vapors. ${ }^{4-6}$ In spite of extensive research in metal NP synthesis, a rationale for the control of the size distribution is still lacking, and this control is more empirical art than science. ${ }^{4,6-8}$

A narrow size distribution is essential for increasing the degree of ordering in NP superlattices. ${ }^{9,10}$ The conditions for narrowing the size distribution when the particles grow under diffusion control were studied by Reiss. ${ }^{11}$ Ostwald ripening and coalescence induce polydispersity in NP synthesis; remarkably, Ostwald ripening does not lead to a thermodynamically stable size. ${ }^{4}$ Colloid-based synthesis of metal NPs limit these two processes by providing a confined environment to carry out the metal reduction reaction. ${ }^{4}$ Thus, colloid-based synthesis enables production of smaller sizes of metal NPs in larger bulk quantities than other techniques. The reverse micelle synthesis ${ }^{2}$ usually produces NPs whose average size depends somewhat on the micelle size, ${ }^{10,12}$ but many surfactants are only weakly bound to the surface of the NPs and they can grow much larger than the micelle size. ${ }^{4}$

Digestive ripening (DR) is an established procedure for preparing narrow size distributions of ligand-stabilized NPs of noble metals, transition metals, lanthanide oxides, metal sulfides and halides, and II-VI semiconductors, as well as alloys and core-shell bimetallic NPs. ${ }^{4-6,9,13-17}$ Most often, it leads to spherical NPs, but not always. ${ }^{18}$ Essentially, DR is a thermochemical step that follows the SMAD, ${ }^{4,5}$ the Turkevich ${ }^{3}$ or the reverse micelle synthesis ${ }^{11,19,20}$ in which a ligandexchange reaction induces the redistribution of atoms from polydisperse NPs and leads to a much narrower size distribution; typically with smaller average size, although the opposite has also been observed. ${ }^{6,21}$ The term DR introduced by Klablunde and co-workers ${ }^{10}$ is often associated with heating the as-prepared, polydisperse colloidal solution in the presence of excess strong capping ligand at or near the boiling point of the solvent under reflux. ${ }^{4,6}$ The process can also take place at room temperature if the concentration of capping ligand is large enough. ${ }^{22-26}$ The choice of ligands is certainly important and thiols, amines, silanes, phosphines, mercaptopropionic and oleic acids, etc., have been used as DR agents. ${ }^{4}$ The DR process is often carried out in toluene or other organic solvents, but aqueous media have also been used. ${ }^{5,13}$

In spite of the experimental efforts to provide insights, $5,21,24$ most authors stress that the mechanisms of the DR process are

Received: May 4, 2017

Revised: June 1, 2017

Published: June 1, 2017 
not yet known. ${ }^{4,10,18,22-25,27-31}$ Hence, there is a pressing need for thermodynamic studies that identify the factors determining the size distribution. Well-established facts are (i) etching-type mechanisms by some capping ligands underlie the DR transformations of noble metal NPs, (ii) the mean of the equilibrium size distribution is specific to each metal-ligand pair, (iii) NPs are the necessary starting material as normal metal powder is not susceptible to DR, and (iv) a large amount of ligand, much in excess than required for a monolayer coverage of the NP surfaces, is required for the DR process. ${ }^{7,10,28}$ From an experimental point of view, conditions such as temperature, ${ }^{21,24,26,32}$ refluxing time, ${ }^{21,24,25,33}$ metalligand interaction, ${ }^{4,24,32,34}$ ligand:metal ratio, ${ }^{2,7,24,25,35-38}$ sequence of reactants, ${ }^{13}$ added surfactant, ${ }^{6}$ etching ions such as bromide, ${ }^{6}$ etc., have an influence on the final size distribution after DR. A recent investigation into the role of oxygen and other radicals in Au NP synthesis strongly suggests that the necessity for oxygen in the thiol-etching of colloidal gold and in the Brust-Schiffrin synthesis arises from the formation of the $\mathrm{O}_{2}$ diradical. $^{39}$

The Gibbs-Thomson equation predicts the existence of a critical size for the growth of NPs, which corresponds to a maximum of the free energy, and hence an unstable NP size. ${ }^{40}$ A significant step forward toward a theory of DR was achieved when the existence of a thermodynamically stable NP size was demonstrated by considering the electrostatic energy in addition to the interfacial energy. ${ }^{41,42}$ However, the major drawback of this theory of DR is that it neglects the role of most important agent in the DR process, the capping ligand. ${ }^{31,34}$ A DR theory that explains the stabilizing role of the ligands and its pivotal role in determining the NP size distribution is missing. ${ }^{4,10,22-25,27-31}$ Moreover, such theory should explain the observations of increasingly smaller NPs produced when the concentration of ligands increases and when ligands with stronger binding energy to the NP surface are used. $^{25}$

The Brust-Schiffrin synthesis of thiol-stabilized Au NPs of $(2 \pm 1) \mathrm{nm}$ diameter provided a striking evidence of the control that the capping ligand exerts on the NP size. ${ }^{2}$ The control of the thiol:Au ratio and the reaction temperature proved to be very effective for synthesizing core sizes ranging from 1.5 to $5 \mathrm{~nm}$. Soon after this landmark in Au NP research, ${ }^{1}$ Gelbart and co-workers presented a statistical thermodynamic theory for the thiol-meditated size control of uncharged $\mathrm{Au}$ NPs. ${ }^{7}$ This theory successfully explained that the mean NP size could be sensitively controlled by varying the thiol:Au mole ratio. These authors also provided experimental data that confirmed the theoretical predictions, and other authors ${ }^{35-38}$ obtained similar results (Figure 1). However, an extension of the theory to incorporate the stabilizing effects of both the chemisorption of the capping ligand and the solvation energy of the charged NPs has remained elusive. In this work, we present the underlying statistical thermodynamics of the DR of ligandstabilized, charged metal NPs. We show that the electrostatic energy is relevant for very small NPs but the capping ligand plays the decisive role in determining the equilibrium size distribution. The theoretical predictions are in qualitative agreement with the experimental observations on the role of the ligand:metal ratio and provide valuable insights on the DR process.

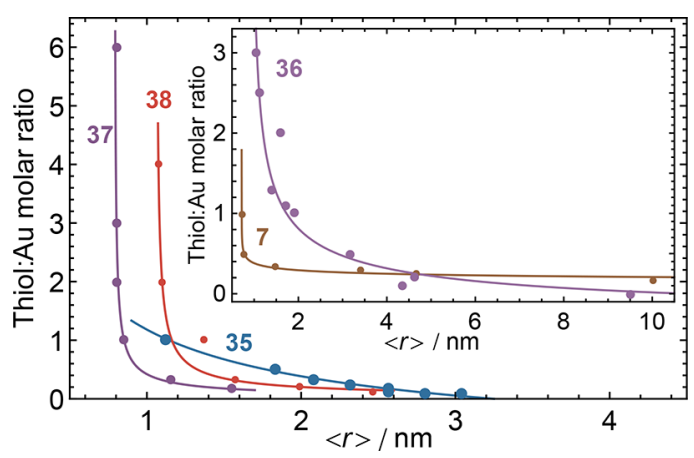

Figure 1. Experimental results of the average NP core radius as determined by the thiol:metal mole ratio during the two-phase BrustSchiffrin synthesis using dodecanethiolate $7,35,37,38$ or 3-mercaptopropionate ligands ${ }^{36}$ (corresponding reference numbers given on the curves).

\section{THEORY}

There is a widespread conviction that the size distributions after the DR process are determined by the thermodynamic stability. ${ }^{5,10,16,23,43,44}$ We present here an equilibrium theory that can enhance our understanding of this process as it generalizes previous models and is consistent with experimental observations. Thermodynamic models are suited to describe the observed NP size distribution, but their predictions should be considered with caution in the case of nanoclusters below $1.5 \mathrm{~nm}$ in diameter, if electronic or geometric shell closings are not considered in the model.

Thermodynamic studies of NP stabilization most often consider spherical geometry instead of fcc polyhedra. Both, theoretical and experimental results indicate that the undercoordinated atoms at edges and corners relax with inward displacements while terrace atoms are displaced outward so that the NPs assume a nearly spherical shape upon relaxation. ${ }^{45}$ In addition, the influence of the adsorbed ligands on the NP structure further supports the spherical approximation the ligand-stabilized NPs. ${ }^{7,46}$ The number of metal atoms and bound ligands in a NP of radius $r_{n}$ are $n \approx \kappa^{3} r_{n}{ }^{3}$ and $L_{n} \approx L_{1} n^{2 / 3}$, respectively, where $\kappa \equiv\left(4 \pi / 3 V_{\mathrm{M}}\right)^{1 / 3}, L_{1} \equiv 4 \pi /\left(\kappa^{2} A_{\mathrm{L}}\right), V_{\mathrm{M}}$ is the volume per metal atom in the NP, and $A_{\mathrm{L}}$ is the area per ligand; i.e. $A_{n}=L_{n} A_{\mathrm{L}} \approx 4 \pi r_{n}^{2}$ is the total NP surface area (see Supporting Information). There is no need to include the slight size dependence of the volume per atom that is observed in very small NPs.

Strong capping ligands play a dual role as stabilizing adsorbates and as efficient etchants. ${ }^{20}$ The chemisorption process induces strong changes in the substrate with the formation of vacancy islands ("etch pits") of monatomic depth in the case of aliphatic thiols and of gold islands of monatomic height ("adatoms") in the case of most of the aromatic thiols. ${ }^{44}$ In the colloidal solution, a distribution of differently sized, monolayer-protected NPs coexists in equilibrium with the free ligands. The ligand-mediated transfer of metal atoms between the NPs allows for establishing the thermodynamic equilibrium and gives meaning to the concept of chemical potential $\mu$ of free atoms in solution. The bulk metal is chosen as the standard state for the metal atoms, and $\mu^{\circ}$ is the corresponding chemical potential. The chemical potential $\mu_{n}$ of a NP with $n$ metal atoms and $L_{n}$ ligands is

$$
\mu_{n}=\mu_{n}^{\circ}+\mu_{n}^{\text {surf }}+\mu_{n}^{\text {bind }}+\mu_{n}^{\text {el }}+k T \ln x_{n}
$$


where $\mu_{n}^{\circ}=n \mu^{\circ}+L_{n} \mu_{\mathrm{L}}^{\circ}$ is the standard chemical potential of the NPs, $\mu_{n}^{\text {surf }}, \mu_{n}^{\text {bind }}$, and $\mu_{n}^{\mathrm{el}}$ are, respectively, the contributions of surface tension (interfacial free energy), ligand chemisorption and electrostatic solvation energy, $x_{n}=N_{n} / N_{\text {tot }}$ and $N_{n}$ are the mole fraction and number of NPs with $n$ metal atoms, and $N_{\text {tot }}$ is the total number of solvent molecules, ligand molecules, NPs and metal atoms in the colloidal solution. Under equilibrium conditions, NPs of different sizes have different probabilities, i.e. different mole fractions $x_{n}(n)$. Solving for $x_{n}(n)$ from eq 1 and using the condition $\mu_{n}=n \mu+L_{n} \mu_{\mathrm{L}}$ that ensures the minimization of the free energy of the colloidal solution over all possible distributions, ${ }^{47}$ the NP size distribution under thermodynamic equilibrium is

$$
x_{n}(n)=\lambda^{n} x_{\mathrm{L}}^{L_{n}} \exp \left[-\left(\mu_{n}^{\text {surf }}+\mu_{n}^{\text {bind }}+\mu_{n}^{\text {el }}\right) / k T\right]
$$

where $\lambda \equiv \exp \left[\left(\mu-\mu^{\circ}\right) / k T\right]$ is the saturation parameter (with respect to bulk metal). The total amount of metal atoms in the colloidal solution, $N_{\mathrm{M}}=N_{\text {tot }} \Sigma_{n} n x_{n}$, determines the chemical potential $\mu$ of the metal atoms. Similarly, the amount of free and bound ligands in the solution, $N_{\mathrm{L}}=N_{\text {tot }}\left(x_{\mathrm{L}}+\sum_{n} L_{n} x_{n}\right)$, increases with increasing mole fraction $x_{\mathrm{L}}=\exp \left[\left(\mu_{\mathrm{L}}-\mu_{\mathrm{L}}^{\circ}\right) / k T\right]$ of free ligands, where $\mu_{\mathrm{L}}$ is their chemical potential. The ligand:metal mole ratio $N_{\mathrm{L}} / N_{\mathrm{M}}$ and the average NP radius $\langle r\rangle=$ $\Sigma_{n} r_{n} x_{n} / \Sigma_{n} x_{n}$ (not counting the monolayer-protecting shell) can be evaluated from $\lambda$ and $x_{\mathrm{L}}$.

The interfacial free energy is a very important characteristic of NPs, as most effects on their physical properties are related to the Gibbs-Thomson equation of nanothermodynamics. ${ }^{40}$ The interfacial free energy contribution to the chemical potential is given by $\mu_{n}^{\text {surf }}=\gamma_{\mathrm{n}} A_{n}$, which simplifies to $\mu_{n}^{\text {surf }}=$ $\gamma_{\infty} 4 \pi r_{n}^{2}$ in the case of very large NPs. Recent molecular dynamics (MD) simulations data ${ }^{43}$ can be accurately fitted to $\mu_{n}^{\text {surf }}=a n^{2 / 3}$ and allow us to calculate $a \approx 4 \pi \gamma_{\infty} / \kappa^{2}=1.8765 \mathrm{eV}$, which corresponds to a surface tension $\gamma_{\infty}=0.980 \mathrm{~J} / \mathrm{m}^{2}$ for very large $\mathrm{Au}$ NPs, in agreement with the experimental values. ${ }^{45}$ The expression $\gamma_{\mathrm{n}} A_{\mathrm{n}} \approx a n^{2 / 3}$ does not imply that the interfacial free energy $\gamma_{n}$ of Au NPs is size independent. On the contrary, $\gamma_{n}$ increases with decreasing NP size because of the associated increase in the fraction of edge and corner sites (see Supporting Information).

Like any other aggregation process, the nucleation and growth of NPs from metal atoms is entropically unfavorable. Disregarding $\mu_{n}^{\text {bind }}$ and $\mu_{n}^{\mathrm{el}}$, supersaturation conditions $\lambda>1$ are required to observe non-negligible values of $x_{n}$ because $\mu_{n}^{\text {surf }}>0$. Then, the function $\ln x_{n}=n \ln \lambda+L_{n} \ln x_{\mathrm{L}}-\mu_{n}^{\text {surf }} / k T$ has a minimum as a function of $n$, so that there is no peak in the size distribution. The NPs with radii larger than the critical one grow continuously at the expense of the dissolution of those with lower radii (Ostwald ripening).

The $\mathrm{Au}$-thiolate particles obtained by DR are either neutral or negatively charged overall. ${ }^{5}$ Charge numbers of around $z=$ -5 have been measured for glutathione-Au hydrophilic NPs with a diameter of $3 \mathrm{~nm} .{ }^{48}$ Actually, the charge on the NP due to excess or deficiency of electrons in the metallic core is determined by its Fermi level equilibration with the solution. ${ }^{49,50}$ The DR theory by Lee et al., ${ }^{41,42}$ does not incorporate the term $\mu_{n}^{\text {bind }}<0$ in eq 2. A minimum of the free energy as a function of the NP radius, i.e., a charge-stabilized NP size appears in the absence of capping ligands because $\mu_{n}^{\text {surf }}$ $>0$ increases with increasing $n$ and $\mu_{n}^{\text {el }}>0$ decreases. Hence, their sum exhibits a minimum for some value of $n$, which depends on the saturation parameter $\lambda$. However, the corresponding NP size distribution is extremely narrowed and peaked over a very small, stable size.

By considering NPs with charge number $z=20$ in vacuum at $120{ }^{\circ} \mathrm{C}$, Lee et al. showed that the stable size was ca. $1.5 \mathrm{~nm}$ in radius. ${ }^{41}$ An alleged, direct experimental verification of this theory was based on the only observation that the size reduction from $(3.7 \pm 1.8)$ to $(2.7 \pm 0.5) \mathrm{nm}$ after $\mathrm{DR}$ of $\mathrm{ZnO}$ NPs with triethanolamine was accompanied by an increase of their zeta potential from +6 to $+21 \mathrm{mV} .{ }^{51}$ However, this theory predicts the opposite trend, i.e., that the stable size increases with increasing NP charge number. Recently, some skepticism has been manifested on the basis that there is no experimental support for the assumption made in this theory that the charge number is independent of the particle size. ${ }^{25,26,33}$ More importantly, for low NP charge numbers in a dielectric solvent, this theory predicts an extremely narrow distribution with such a small average size that electronic and geometric shell closing effects on nanocluster stability would have to be taken into account.

For the sake of comparison with the DR theory by Lee et al., ${ }^{41}$ the contribution of the electrostatic solvation energy to $\mu_{n}$ is estimated as $\mu_{n}^{\mathrm{el}}=z^{2} e^{2} /\left(8 \pi \varepsilon_{0} \varepsilon_{\mathrm{r}} r_{n}\right)$, where $\varepsilon_{\mathrm{r}}$ is the relative electrical permittivity. This expression applies when the ligand chains have no charged functionalities and there are no adsorbed ionic surfactants or specifically adsorbed ions (e.g., bromide ions). In addition, this expression of $\mu_{n}^{\mathrm{el}}$ does not take into account the ionic cloud that surrounds a charged NP in an electrolyte solution; ${ }^{52}$ which would contribute with an additional factor $1 /\left(1+r_{n} / L_{\mathrm{D}}\right)$, where $L_{\mathrm{D}}$ is the Debye length of the colloidal solution.

Although the existence of a stable size is very interesting, it should be emphasized that DR is based on the use of stabilizing ligands and that the electrostatic stabilization of the NPs synthesized by the Brust-Schiffrin method is due to the ionic surfactants employed and the electrical double layers around the thiol-metal NPs. ${ }^{53}$ None of these aspects is considered in the DR theory by Lee et al. ${ }^{41,42}$

The contribution of ligand chemisorption can be estimated as $\mu_{n}^{\text {bind }}=-E_{n} L_{n} \approx-E_{\infty} L_{1} n^{2 / 3}+c$ where $E_{\mathrm{n}}=E_{\infty}-k T A_{\mathrm{E}} / r_{n}{ }^{2}$ is the binding energy per ligand and $c=4 \pi k T A_{\mathrm{E}} / A_{\mathrm{L}}$. The term $k T A_{\mathrm{E}} / r_{n}{ }^{2}$ accounts for the bending energy of the ligand monolayer, ${ }^{7}$ and describes the preference of the capping ligand for a less curved surface, as observed in DR studies. ${ }^{27}$ Under isothermal conditions, the constant $c$ is practically irrelevant for the NP size distribution.

Both, $\mu_{n}^{\text {surf }}$ and $\mu_{n}^{\text {bind }}$ are essentially proportional to the NP surface area, $\mu_{n}^{\text {surf }}+\mu_{n}^{\text {bind }}=a^{\prime} n^{2 / 3}+c$. It is often stated that the chemisorption of capping ligands lowers the interfacial free energy of the metal NPs because $\mu_{n}^{\text {bind }}<0 .{ }^{46,54}$ Indeed, the effective interfacial free energy is $\gamma_{\text {eff }}=a^{\prime} / L_{1} A_{\mathrm{L}}=\gamma_{\infty}-E_{\infty} / A_{\mathrm{L}}$. The decreased thermal stability of NPs with respect to their partner bulk materials, as reflected for instance in their decreased melting temperature, is due to the free energy of the NP surface. ${ }^{40}$ The reduction $\gamma_{\text {eff }}-\gamma_{\infty}=-E_{\infty} / A_{\mathrm{L}}<0$ in the effective interfacial free energy leads to an increased thermal stability of the NPs induced by the capping ligands. ${ }^{54}$

Large metal-ligand binding energies are involved during the DR process, as evidenced by the fact that the as-prepared metal NPs are etched by thiols. ${ }^{4,24}$ The ligand stabilization of NPs in solution requires a sufficiently large binding energy, and this implies that $\gamma_{\text {eff }}$ and $a^{\prime}$ must be negative. ${ }^{7}$ (This point marks the difference between theoretical models including or not the effect of the ligand.) Nucleation can then occur in under- 
saturation conditions, ${ }^{7} \lambda<1$ and $1>x_{\mathrm{L}}>\exp \left(\gamma_{\mathrm{eff}} A_{\mathrm{L}} / k T\right)$. In the case of uncharged NPs, $\ln x_{n}=n \ln \lambda+L_{n} \ln x_{\mathrm{L}}-\left(a^{\prime} n^{2 / 3}+c\right) /$ $k T$ has a maximum as a function of $n$ that describes the most likely, stable NP size. In the case of charged particles, $\mu_{n}^{\mathrm{el}}>0$ reduces the mole fraction $x_{n}$ of the smaller NPs (as the solvation energy is non negligible only for small NPs) and, hence, shifts the distribution peak toward larger radii.

The NP size distribution is described by eq $2,-k T \ln x_{\mathrm{n}}=\mu_{n}^{\mathrm{el}}$ $+\mu_{n}^{\text {surf }}+\mu_{n}^{\text {bind }}-L_{n} k T \ln x_{\mathrm{L}}-n k T \ln \lambda$ (Figure 2). The term $\mu_{n}^{\mathrm{el}}$ scales as $n^{-1 / 3}$, the sum $\mu_{n}^{\text {surf }}+\mu_{n}^{\text {bind }}-L_{n} k T \ln x_{\mathrm{L}}-c$ scales as $n^{2 / 3}$ and the term $n k T \ln \lambda$ obviously scales as $n$. The sum of these

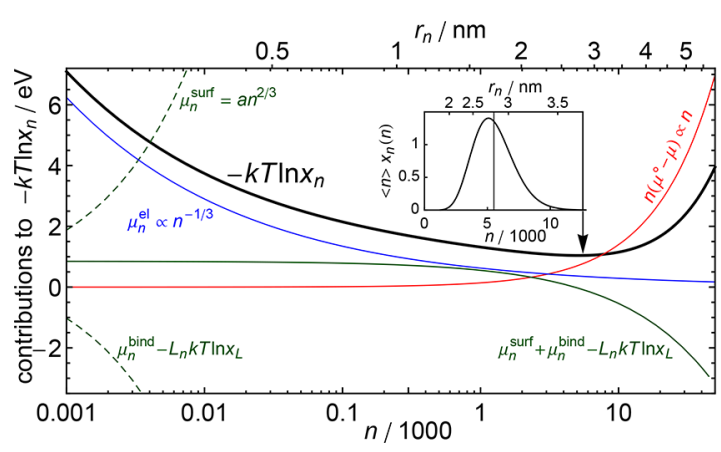

Figure 2. Arrow marks the minimum of $-k T \ln x_{n}(n)$ (thick black curve) which corresponds to the mode of the equilibrium size distribution $x_{n}(n)$ (eq 2 and inset). The function $-k T \ln x_{n}$ is the sum of a contribution $\mu_{n}^{\text {el }}$ scaling as $n^{-1 / 3}$ (blue), a contribution $\mu_{n}^{\text {surf }}+\mu_{n}^{\text {bind }}$ $-L_{n} k \operatorname{Tin} x_{\mathrm{L}}$ that is the sum of the constant $c$ and a term scaling as $n^{2 / 3}$ (solid green, sum of the two dashed green curves), and the contribution $n\left(\mu^{\circ}-\mu\right)$ (red). Parameter values are $120{ }^{\circ} \mathrm{C}, z= \pm$ $5, \varepsilon_{\mathrm{r}}=18.11,1-\lambda=0.004115$, and $x_{\mathrm{L}}=6.3544 \times 10^{-8}$; others given in the main text. The metal:ligand ratio is then equal to 6.06 and the average radius is $\langle r\rangle=2.79 \mathrm{~nm}$ (vertical line at $\langle n\rangle=5519$ in inset). contributions shows a minimum for some value of $n$. It corresponds to the peak of the size distribution $x_{n}(n)$ (Figure 2 inset), but not to a peak of the chemical potential $\mu_{n}(n)$. Indeed, as $\exp \left[\left(\mu_{n}-\mu_{n}^{\circ}\right) / k T\right]=\lambda^{n} x_{L}^{L_{n}}, \mu_{n}(n)$ is a monotonously decreasing function of $n$ when $\lambda<1$ and $x_{\mathrm{L}}<1$. The conditions for observing the typical equilibrium size distributions are $\lambda \rightarrow$ $1-$ and $x_{\mathrm{L}} \rightarrow \exp \left(\gamma_{\mathrm{eff}} A_{\mathrm{L}} / k T\right)+$, so that the coefficients of the terms that scale as $n^{2 / 3}$ and $n$ are both small.

Our theoretical model, eq 2, satisfactorily describes the experimental observations of the variation of the average size (Figure 1) and the width of the size distribution, as well as the influence of the relevant DR parameters. In particular, parts a$\mathrm{d}$ of Figure 3 analyze the influence of the solvent dielectric constant, the NP charge number, the ligand binding energy, the ripening temperature and the ligand chain length. These figures have in common the leftmost curve, which is taken as a reference. It corresponds to uncharged NPs with the density of bulk Au $V_{\mathrm{M}}=0.01695 \mathrm{~nm}^{3,},{ }^{5,7,41}$ the interfacial free energy $\gamma_{\infty}=$ $0.980 \mathrm{~J} / \mathrm{m}^{2}$ as determined from recent MD simulations of $\mathrm{Au}$ $\mathrm{NPs}^{45}$ (equivalent to $\gamma_{n} A_{n} \approx a n^{2 / 3}$ with $a=1.8765 \mathrm{eV}$, see Supporting Information), the dodecanethiol binding area $A_{\mathrm{L}}=$ $0.1764 \mathrm{~nm}^{2}$ as determined from experimental data ${ }^{5,7,10}$ (see Supporting Information), a binding energy per ligand $E_{n}=1.60$ $\mathrm{eV}$ estimated from experimental observations (see Supporting Information), and a temperature of $120{ }^{\circ} \mathrm{C}$ commonly used for DR with boiling solvent or silicon oil baths. ${ }^{22,41}$ In other words, the reference curve includes no fitting parameter but only values estimated from recent publications. The capping ligand:metal mole ratio vs average radius plots (Figure 3) clearly show that no further narrowing of the size distribution is observed above a certain ligand:metal ratio, ${ }^{25}$ as the minimum size is determined by the thermodynamic stability of the capped NPs. ${ }^{7}$ When observed in the metal:ligand mole ratio vs average radius plot (insets of Figure $3 \mathrm{~b}-\mathrm{d}$ ), the reference curve is fully
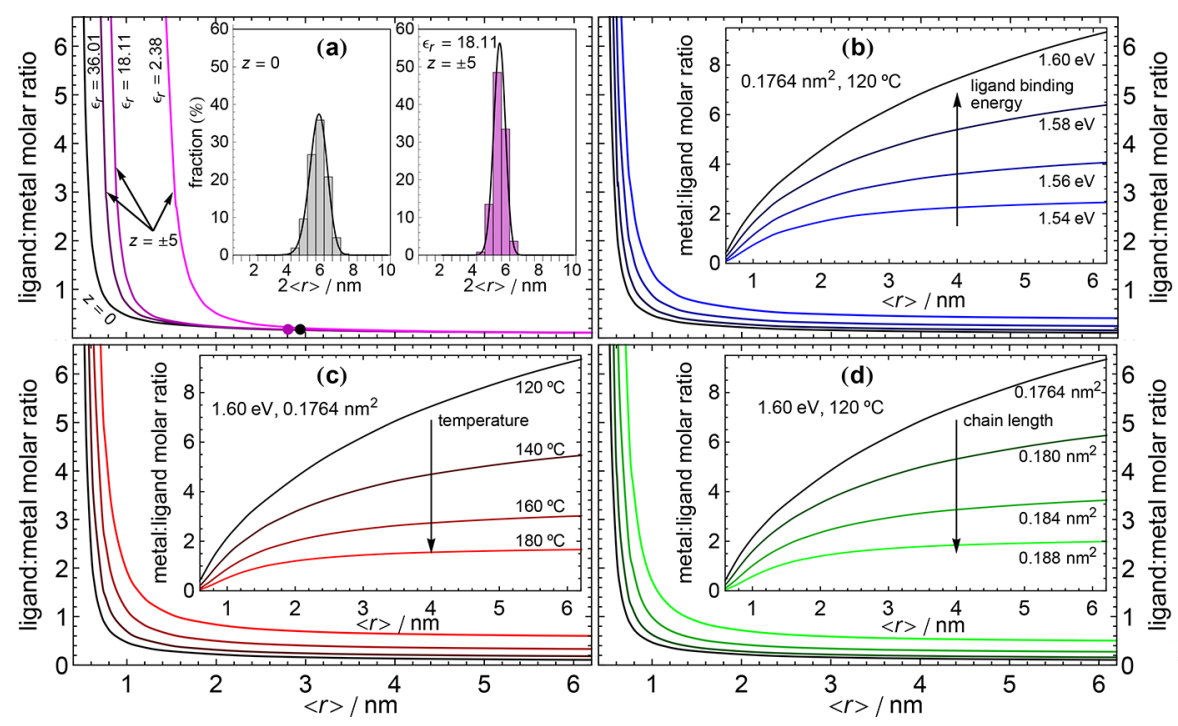

Figure 3. Average NP radius decreases with increasing thiol:metal mole ratio. The equilibrium distribution results from two opposing tendencies: the average size tends to increase in order to reduce the interfacial free energy and tends to decrease in order to increase the number of thiol-metal bonds. (a) For fixed $A_{\mathrm{L}}=0.1764 \mathrm{~nm}^{2}, E_{n}=1.60 \mathrm{eV}$ and $120^{\circ} \mathrm{C}$, the effect of the NP charge is shown by considering the relative permittivities of typical DR solvents: toluene $\left(\varepsilon_{\mathrm{r}}=2.38\right)$, 2-butanone $\left(\varepsilon_{\mathrm{r}}=18.11\right)$ and acetonitrile $\left(\varepsilon_{\mathrm{r}}=36.01\right)$. In addition to shifting the distribution toward larger cores, the NP charge also narrows the distribution (the insets correspond to the two points with $\langle r\rangle \approx 3 \mathrm{~nm}$ marked on the curves). (b and c) For fixed metal:ligand ratio, the average core size increases with increasing ripening temperature and with decreasing strength of the metal-ligand interaction. (d) For fixed metal:ligand ratio, larger chain lengths slightly reduce the ligand packing density (larger $A_{\mathrm{L}}$ ) and shift the average size toward larger values. 
consistent with that derived by Gelbart et al. (see Figure 2 of ref 7 ), except for quantitative changes due to the different parameter values used.

\section{RESULTS AND DISCUSSION}

Effect of Charge on the Particle Size Distribution. The $\mathrm{Au}$-thiolate particles obtained by DR are either neutral or bear a small negative charge. ${ }^{5,27,48}$ The DR process is often carried out in solvents with low relative permittivity, such as toluene $\left(\varepsilon_{\mathrm{r}}\right.$ $=2.38)$ and 2-butanone $\left(\varepsilon_{\mathrm{r}}=18.11\right)$. The effect of the NP charge of the equilibrium size distribution is 2-fold: it increases the average radius (Figure 3a) and narrows the size distribution (Figure 3a insets). In any case, the effect of the NP charge is less noticeable for larger sizes, as it should be expected from the $1 / r$ dependence of the electrostatic energy. One of the questions that have intrigued scientists acquainted with the DR process is that when using very high ligand:metal ratio and boiling toluene, all the particles become similar in size, with average diameters typically in the range $4-5 \mathrm{~nm} .{ }^{55}$ The thermodynamic theory presented above clearly predicts a relatively narrow equilibrium size distribution, and hence, it explains why the particles become similar in size. Moreover, the observed diameters are consistent with the predictions of Figure 3 for low relative permittivity solvents such as toluene and 2-butanone. In any case, the range $4-5 \mathrm{~nm}$ mentioned above should be considered a typical one, as the DR process can lead to both larger and smaller sizes (e.g., when surfactants are used in addition to the capping ligands). ${ }^{6}$

Effect of Ligand on the Particle Size Distribution. The capping ligand exerts a key role in the DR process. Stronger binding ligands give rise to smaller average sizes, while weaker ligands, such as amines, lead to larger sizes. ${ }^{14}$ For fixed metal:ligand ratio, the thermodynamic equilibrium of ligandstabilized NPs predicts that a decrease in binding energy increases the average size (Figure $3 \mathrm{~b}$ inset), in agreement with the experimental observations. ${ }^{4,24,32}$ Moreover, the effect of the binding energy described in Figure $3 \mathrm{~b}$ also helps to understand the observations for dodecanethiol-stabilized $\mathrm{Ag}, \mathrm{Au}$, and $\mathrm{Pd}$ NPs. Thiol possess the strongest attraction for Ag, intermediate for $\mathrm{Au}$ and the weakest for Pd. For fixed metal:ligand ratio, the theoretical prediction (Figure $3 \mathrm{~b}$ inset) agrees with experimental observations: smaller average size for Ag NPs (higher binding energy), intermediate for Au NPs and larger for Pd NPs. ${ }^{34}$

The influence of the chain length of the capping ligands is not as important as the other factors mentioned above, but experimental observations indicate that larger chain lengths lead to larger cores after the DR process. For example, Prasad et al. studied the DR of Au NPs in toluene using the series $\mathrm{C}_{8} \mathrm{H}_{17} \mathrm{SH}$, $\mathrm{C}_{10} \mathrm{H}_{21} \mathrm{SH}, \mathrm{C}_{12} \mathrm{H}_{25} \mathrm{SH}$, and $\mathrm{C}_{16} \mathrm{H}_{33} \mathrm{SH}$ of stabilizing ligands and observed that respective average diameters were $4.5,4.7,4.7$, and $5.5 \mathrm{~nm} .{ }^{27}$ This trend has been confirmed in later studies for both alkanethiols and alkylisothiocyanates. ${ }^{32,56}$ With amines, the tendency is not observed so clearly. Using dodecylamine, hexadecylamine, and octadecylamine as (relatively weak) capping ligands of Au NPs, Jagirdar and co-workers observed average diameters in the ranges 10-12, 7.5-9.5, and 10-13 $\mathrm{nm}$, respectively. ${ }^{24}$ The chain length affects the packing density of the ligand ${ }^{57}$ and can be described as an increase of the effective ligand area $A_{\mathrm{L}}$ (lower packing density) with increasing chain length. Considering the experimentally observed variation of $A_{\mathrm{L}}$ with the chain length, the thermodynamic theory also predicts that the average NP size increases with increasing chain length for constant metal:ligand ratio (Figure $3 \mathrm{~d}$ inset).

Effect of Temperature on the Particle Size Distribution. The ripening temperature affects the final size distribution, with higher temperatures producing larger NPs. ${ }^{21,32}$ Some authors discuss these observations in terms of the size dependence of the NP melting temperature. ${ }^{13}$ Our point is that the equilibrium size distribution is determined, among other parameters, by temperature. The width of the distribution is also observed to increase with temperature, at least for some concentrations of dodecanethiol. ${ }^{26}$ Similarly, for NPs in solid state Teranishi et al. found that the heat treatment of dodecanethiol-stabilized $\mathrm{Au}$ NPs increased the mean diameter from $3.4 \mathrm{~nm}$ at $150{ }^{\circ} \mathrm{C}$, to $5.4 \mathrm{~nm}$ at $190{ }^{\circ} \mathrm{C}$, and further to $6.8 \mathrm{~nm}$ at $230{ }^{\circ} \mathrm{C} .{ }^{58}$ With typical parameter values used in the experiments (i.e., no fitting parameters), the thermodynamic equilibrium for ligand-stabilized NPs predicts that the average size increases with temperature for constant metal:ligand ratio (Figure $3 \mathrm{c}$ inset), in agreement with the experimental observations. ${ }^{21,26,32}$

\section{CONCLUSIONS}

In spite of the experimental efforts to provide insights on $\mathrm{DR},{ }^{5,21,24}$ many authors stress that the DR process is still poorly understood. ${ }^{4,10,18,22-25,27-31}$ The DR studies often cite the theory proposed by Lee et al. $^{41}$ which considers the electrostatic energy of charged NPs as one of the key contributions. However, the DR studies also consider that this theory is not satisfactory as it neglects the role of the DR agent, the capping ligand. ${ }^{31,34}$ Gelbart and co-workers presented a statistical thermodynamic theory of thiol-mediated size control of uncharged $\mathrm{Au}$ NPs that explained how the average value of the NP size distribution could be sensitively controlled by varying the thiol:Au mole ratio, in agreement with the experimental observation by Schiffrin et al. as well as their own. ${ }^{7}$ In order to better understand the different factors involved in the DR process, it seems necessary to generalize the theories by Lee et al. ${ }^{41}$ and Gelbart and co-workers ${ }^{7}$ to include the effects of both NP charge and binding of the stabilizing ligand; as well as to make it more comprehensible by explaining its foundations. We have presented the underlying statistical thermodynamics of the DR of ligand-stabilized, charged metal NPs and showed that the theoretical predictions are in qualitative agreement with the experimental observations, not only on the role of the metal:ligand ratio but also on the effects of the NP charge, the relative permittivity of the solvent, the ripening temperature, the binding energy, and the ligand chain length (Table 1).

Although thermodynamics does not clarify mechanistic details, it certainly provides valuable insights on the DR process. The theoretical predictions made in this study only

Table 1. Summary of the Effects of Increasing One Parameter on the Average Particle Radius

$\begin{array}{lc}\text { increasing parameter } & \text { effect on }\langle r\rangle \\ \text { ligand:metal mole ratio } & \downarrow \\ \text { absolute particle charge } & \uparrow \\ \text { relative permittivity } & \downarrow \\ \text { ligand binding energy } & \downarrow \\ \text { ligand chain length } & \uparrow \\ \text { temperature } & \uparrow\end{array}$


make use of parameters determined by recent experimental and simulation studies of the systems under consideration. It is then remarkable that a relatively simple theory with no fitting parameters describes so satisfactorily the different factors experimentally influencing the DR process. Our theoretical model is compatible with and extends previous models, while remaining simple and clear. Additional effects might be incorporated but at the expense of increasing complexity. For example, more refined models might be used for evaluating the electrostatic energy, ${ }^{59}$ the probability distribution in eq 2 might include a factor describing the influence of mass on the translational and rotational partition functions of the NPs, ${ }^{60}$ and that Hill's subdivision potential might be taken into account to slightly modify eq $1 .^{40}$

\section{ASSOCIATED CONTENT}

\section{S Supporting Information}

The Supporting Information is available free of charge on the ACS Publications website at DOI: 10.1021/acs.jpcc.7b04234.

Ligand packing density on Au NPs, surface energy and size dependence of the surface tension of $\mathrm{Au}$ NPs, and alkanethiol binding energy on Au NPs (PDF)

\section{AUTHOR INFORMATION}

\section{Corresponding Author}

*(J.A.M.) Telephone: +34-963544478. E-mail: jose.a. manzanares@uv.es.

\section{ORCID}

José A. Manzanares: 0000-0002-5402-6842

Pekka Peljo: 0000-0002-1229-2261

Hubert H. Girault: 0000-0001-5573-5774

\section{Notes}

The authors declare no competing financial interest.

\section{ACKNOWLEDGMENTS}

J.A.M. acknowledges financial support from the Ministerio de Economía y Competitividad and European Regional Development Fund through project MAT2015-65011-P and thanks the University of Valencia for a grant-in-aid to visit EPFL Valais Wallis. P.P. and H.H.G. acknowledge support from the Swiss National Science Foundation (Grants Ambizione Energy 160553 and Solar Fuels 2000-20 152 557/1). The authors dedicate this work to the late Professors Howard Reiss and Kyösti Kontturi.

\section{REFERENCES}

(1) Liz-Marzán, L. M. Gold Nanoparticle Research Before and After the Brust-Schiffrin Method. Chem. Commun. 2013, 49, 16-18.

(2) Brust, M.; Walker, M.; Bethell, D.; Schiffrin, D. J.; Whyman, R. Synthesis of Thiol-derivatised Gold Nanoparticles in a Two-phase Liquid-Liquid System. J. Chem. Soc., Chem. Commun. 1994, 0, 801802.

(3) Uppal, M. A.; Kafizas, A.; Lim, T. H.; Parkin, I. P. The Extended Time Evolution Size Decrease of Gold Nanoparticles Formed by the Turkevich Method. New J. Chem. 2010, 34, 1401-1407.

(4) Klabunde, K. J.; Sorensen, C. M.; Stoeva, S. I.; Prasad, B. L. V.; Smetana, A. B.; Lin, X. M. Digestive Ripening, or "Nanomachining," to Achieve Nanocrystal Size Control. In Metal Nanoclusters in Catalysis and Materials Science: The Issue of Size Control; Corain, B., Schmid, G., Toshima, N., Eds.; Elsevier Science: Amsterdam, 2008; pp 233-252.

(5) Jose, D.; Matthiesen, J. E.; Parsons, C.; Sorensen, C. M.; Klabunde, K. J. Size Focusing of Nanoparticles by Thermodynamic
Control through Ligand Interactions. Molecular Clusters Compared with Nanoparticles of Metals. J. Phys. Chem. Lett. 2012, 3, 885-890.

(6) Seth, J.; Prasad, B. L. V. Bromide Ion Mediated Modification to Digestive Ripening Process: Preparation of Ultra-small Pd, Pt, Rh and Ru Nanoparticles. Nano Res. 2016, 9, 2007-2017.

(7) Leff, D. V.; Ohara, P. C.; Heath, J. R.; Gelbart, W. M. Thermodynamic Control of Gold Nanocrystal Size: Experiment and Theory. J. Phys. Chem. 1995, 99, 7036-7041.

(8) Wang, F.; Richards, V. N.; Shields, S. P.; Buhro, W. E. Kinetics and Mechanisms of Aggregative Nanocrystal Growth. Chem. Mater. 2014, 26, 5-21.

(9) Pichler, S.; Bodnarchuk, M. I.; Kovalenko, M. V.; Yarema, M.; Springholz, G.; Talapin, D. V.; Heiss, W. Evaluation of Ordering in Single-Component and Binary Nanocrystal Superlattices by Analysis of Their Autocorrelation Functions. ACS Nano 2011, 5, 1703-1712.

(10) Lin, X. M.; Sorensen, C. M.; Klabunde, K. J. Digestive Ripening, Nanophase Segregation and Superlattice Formation in Gold Nanocrystal Colloids. J. Nanopart. Res. 2000, 2, 157-164.

(11) Reiss, H. The Growth of Uniform Colloidal Dispersions. J. Chem. Phys. 1951, 19, 482-487.

(12) Zhi Fu, Y.; Kou Du, Y.; Yang, P.; Ru Li, J.; Jiang, L. SizeControlled Synthesis of Highly Monodisperse Gold Nanoparticles without a Size-Selection and Long Range Ordered 2-D Arrangement. J. Dispersion Sci. Technol. 2007, 28, 301-307.

(13) Goubet, N.; Ding, Y.; Brust, M.; Wang, Z. L.; Pileni, M.-P. A Way To Control the Gold Nanocrystals Size: Using Seeds with Different Sizes and Subjecting Them to Mild Annealing. ACS Nano 2009, 3, 3622-3628.

(14) Zhang, Q.; Xie, J.; Yang, J.; Lee, J. Y. A Way To Control the Gold Nanocrystals Size: Using Seeds with Different Sizes and Subjecting Them to Mild Annealing. ACS Nano 2009, 3, 139-148.

(15) Wen, T.; Majetich, S. A. Ultra-Large-Area Self-Assembled Monolayers of Nanoparticles. ACS Nano 2011, 5, 8868-8876.

(16) Chen, T.; Yao, Q.; Yuan, X.; Nasaruddin, R. R.; Xie, J. Heating or Cooling: Temperature Effects on the Synthesis of Atomically Precise Gold Nanoclusters. J. Phys. Chem. C 2017, 121, 10743-10751.

(17) Andrieux-Ledier, A.; Tremblay, B.; Courty, A. Synthesis of Silver Nanoparticles Using Different Silver Phosphine Precuersors: Formation Mechanism and Size Control. J. Phys. Chem. C 2013, 117, 14850-14857.

(18) Jeong, J.; Kim, N.; Kim, M.-G.; Kim, W. Generic Synthetic Route to Monodisperse Sub-10 nm Lanthanide Oxide Nanodisks: A Modified Digestive Ripening Process. Chem. Mater. 2016, 28, 172179 .

(19) Schaaff, T. G.; Shafigullin, M. N.; Khoury, J. T.; Vezmar, I.; Whetten, R. L. Properties of a Ubiquitous $29 \mathrm{kDa} \mathrm{Au}: \mathrm{SR}$ Cluster Compound. J. Phys. Chem. B 2001, 105, 8785-8796.

(20) Schaaff, T. G.; Whetten, R. L. Controlled Etching of Au:SR Cluster Compounds. J. Phys. Chem. B 1999, 103, 9394-9396.

(21) Sahu, P.; Prasad, B. L. V. Time and Temperature Effects on the Digestive Ripening of Gold Nanoparticles: Is There a Crossover from Digestive Ripening to Ostwald Ripening? Langmuir 2014, 30, 1014310150.

(22) Kalidindi, S. B.; Jagirdar, B. R. Highly Monodisperse Colloidal Magnesium Nanoparticles by Room Temperature Digestive Ripening. Inorg. Chem. 2009, 48, 4524-4529.

(23) Shankar, R.; Wu, B. B.; Bigioni, T. P. Wet Chemical Synthesis of Monodisperse Colloidal Silver Nanocrystals Using Digestive Ripening. J. Phys. Chem. C 2010, 114, 15916-15923.

(24) Bhaskar, S. P.; Vijayan, M.; Jagirdar, B. R. Size Modulation of Colloidal $\mathrm{Au}$ Nanoparticles via Digestive Ripening in Conjunction with a Solvated Metal Atom Dispersion Method: An Insight Into Mechanism. J. Phys. Chem. C 2014, 118, 18214-18225.

(25) Lin, M.-L.; Yang, F.; Lee, S. Digestive Ripening for Self-assembly of Thiol-capped Gold Nanoparticles: The Effects of Adding Dodecanethiol and Reflux-heating. Colloids Surf., A 2014, 448, 16-22.

(26) Su, Y.-Y.; Yang, F.; Lee, S. Digestive Ripening of Thiol-capped Gold Nanoparticles: Effects of Temperature and Electric Field. Mater. Res. Express 2015, 2, 055007. 
(27) Prasad, B. L. V.; Stoeva, S. I.; Sorensen, C. M.; Klabunde, K. J. Digestive Ripening of Thiolated Gold Nanoparticles: The Effect of Alkyl Chain Length. Langmuir 2002, 18, 7515-7520.

(28) Smetana, A. B.; Klabunde, K. J.; Sorensen, C. M.; Ponce, A. A.; Mwale, B. Low-Temperature Metallic Alloying of Copper and Silver Nanoparticles with Gold Nanoparticles through Digestive Ripening. J. Phys. Chem. B 2006, 110, 2155-2158.

(29) Jose, D.; Jagirdar, B. R. Au@Pd Core-Shell Nanoparticles through Digestive Ripening. J. Phys. Chem. C 2008, 112, 1008910094.

(30) Kalidindi, S. B.; Jagirdar, B. R. Synthesis of Cu@ZnO Core-Shell Nanocomposite through Digestive Ripening of $\mathrm{Cu}$ and $\mathrm{Zn}$ Nanoparticles. J. Phys. Chem. C 2008, 112, 4042-4208.

(31) Arora, N.; Jagirdar, B. R.; Klabunde, K. J. Digestive Ripening Facilitated Atomic Diffusion at Nanosize Regime: Case of $\mathrm{AuIn}_{2}$ and $\mathrm{Ag}_{3}$ In Intermetallic Nanoparticles. J. Alloys Compd. 2014, 610, 35-44.

(32) Sahu, P.; Prasad, B. L. V. Fine Control of Nanoparticle Sizes and Size Distributions: Temperature and Ligand Effects on the Digestive Ripening Process. Nanoscale 2013, 5, 1768-1771.

(33) Lin, M.-L.; Yang, F.; Peng, J. S.; Lee, S. Field Effect on Digestive Ripening of Thiol-capped Gold Nanoparticles. J. Appl. Phys. 2014, 115, 054312 .

(34) Sahu, P.; Prasad, B. L. V. Effect of Digestive Ripening Agent on Nanoparticle Size in the Digestive Ripening Process. Chem. Phys. Lett. 2012, 525-526, 101-104.

(35) Hostetler, M. J.; Wingate, J. E.; Zhong, C.-J.; Harris, J. E.; Vachet, R. W.; Clark, M. R.; Londono, J. D.; Green, S. J.; Stokes, J. J.; Wignall, G. D.; Glish, G. L.; Porter, M. D.; Evans, N. E.; Murray, R. W. Alkanethiolate Gold Cluster Molecules with Core Diameters from 1.5 to $5.2 \mathrm{~nm}$ : Core and Monolayer Properties as a Function of Core Size. Langmuir 1998, 14, 17-30.

(36) Yonezawa, T.; Kunitake, T. Practical Preparation of Anionic Mercapto Ligand-stabilized Gold Nanoparticles and their Immobilization. Colloids Surf., A 1999, 149, 193-199.

(37) Frenkel, A. I.; Nemzer, S.; Pister, I.; Soussan, L.; Harris, T.; Sun, Y.; Rafailovich, M. H. Size-controlled Synthesis and Characterization of Thiol-stabilized Gold Nanoparticles. J. Chem. Phys. 2005, 123, 184701.

(38) Chen, S.-H.; Wang, D.-C.; Chen, G.-Y.; Jan, C.-L. Size Control of the Self-Assembly Gold Nanoparticles. J. Med. Biol. Engn. 2006, 26, $137-142$.

(39) Dreier, T. A.; Ackerson, C. J. Radicals Are Required for Thiol Etching of Gold Particles. Angew. Chem., Int. Ed. 2015, 54, 9249-9252.

(40) García-Morales, V.; Cervera, J.; Manzanares, J. A. Nanothermodynamics. In Handbook of Nanophysics. Principles and Methods; Sattler, K. D., Ed.; CRC Press: Boca Raton, FL, 2011, ch 15.

(41) Lee, D.-K.; Park, S.-I.; Lee, J. K.; Hwang, N.-M. A Theoretical Model for Digestive Ripening. Acta Mater. 2007, 55, 5281-5288.

(42) Hwang, N.-M.; Jung, J.-S.; Lee, D.-K. Thermodynamics and Kinetics in the Synthesis of Monodisperse Nanoparticles. In Thermodynamics - Fundamentals and Its Application in Science; Morales-Rodriguez, R., Ed.; InTech: 2012; pp 371-388.

(43) Ji, Y.; Yang, S.; Guo, S.; Song, X.; Ding, B.; Yang, Z. Bimetallic $\mathrm{Ag} / \mathrm{Au}$ Nanoparticles: A Low Temperature Ripening Strategy in Aqueous Solution. Colloids Surf., A 2010, 372, 204-209.

(44) Pensa, E.; Cortés, E.; Corthey, G.; Carro, P.; Vericat, C.; Fonticelli, M. H.; Benítez, G.; Rubert, A. A.; Salvarezza, R. C. The Chemistry of the Sulfur-Gold Interface: In Search of a Unified Model. Acc. Chem. Res. 2012, 45, 1183-1192.

(45) Ali, S.; Myasnichenko, V. S.; Neyts, E. C. Size-dependent Strain and Surface Energies of Gold Nanoclusters. Phys. Chem. Chem. Phys. 2016, 18, 792-800.

(46) Love, J. C.; Estroff, L. A.; Kriebel, J. K.; Nuzzo, R. G.; Whitesides, G. M. Self-Assembled Monolayers of Thiolates on Metals as a Form of Nanotechnology. Chem. Rev. 2005, 105, 1103-1169.

(47) Ben-Shaul, A.; Gelbart, W. M. Statistical Thermodynamics of Amphiphile Self-Assembly: Structure and Phase Transitions in Micellar Solutions. In Micelles, Membranes, Microemulsions, and
Monolayers; Gelbart, W. M.; Ben-Shaul, A.; Roux, D., Eds.; SpringerVerlag: New York, 1994; pp 1-104.

(48) Lu, Y.; Wang, L.; Chen, D.; Wang, G. Determination of the Concentration and the Average Number of Gold Atoms in a Gold Nanoparticle by Osmotic Pressure. Langmuir 2012, 28, 9282-9287.

(49) Scanlon, M. D.; Peljo, P.; Méndez, M. A.; Smirnov, E.; Girault, H. H. Charging and Discharging at the Nanoscale: Fermi Level Equilibration of Metallic Nanoparticles. Chem. Sci. 2015, 6, 27052720.

(50) Peljo, P.; Manzanares, J. A.; Girault, H. H. Contact Potentials, Fermi Level Equilibration, and Surface Charging. Langmuir 2016, 32, $5765-5775$

(51) Jacob, N. M.; Thomas, T. Digestive Ripening and Green Synthesis of Ultra-small $(r<2 \mathrm{~nm})$ Stable $\mathrm{ZnO}$ Quantum Dots. Ceram. Int. 2014, 40, 13945-13952.

(52) Peljo, P.; Manzanares, J. A.; Girault, H. H. Variation of the Fermi level and the Electrostatic Force of a Metallic Nanoparticle upon Colliding with an Electrode. Chem. Sci. 2017, DOI: 10.1039/ C7SC00848A.

(53) Waters, C. A.; Mills, A. J.; Johnson, K. A.; Schiffrin, D. J. Purification of Dodecanethiol Derivatised Gold Nanoparticles. Chem. Commun. 2003, 540-541.

(54) Liang, L. H.; Shen, C. M.; Du, S. X.; Liu, W. M.; Xie, X. C.; Gao, H. J. Increase in Thermal Stability Induced by Organic Coatings on Nanoparticles. Phys. Rev. B: Condens. Matter Mater. Phys. 2004, 70, 205419.

(55) Stoeva, S.; Klabunde, K. J.; Sorensen, C. M.; Dragieva, I. GramScale Synthesis of Monodisperse Gold Colloids by the Solvated Metal Atom Dispersion Method and Digestive Ripening and Their Organization into Two- and Three-Dimensional Structures. J. Am. Chem. Soc. 2002, 124, 2305-2311.

(56) Kuo, C.-T.; Yu, J.-Y.; Huang, M.-J.; Chen, C.-H. On the Size Evolution of Gold-Monolayer-Protected Clusters by Ligand PlaceExchange Reactions: The Effect of Headgroup-Gold Interactions. Langmuir 2010, 26, 6149-6153.

(57) Hinterwirth, H.; Kappel, S.; Waitz, T.; Prohaska, T.; Lindner, W.; Lämmerhofer, M. Quantifying Thiol Ligand Density of SelfAssembled Monolayers on Gold Nanoparticles by Inductively Coupled Plasma-Mass Spectrometry. ACS Nano 2013, 7, 1129-1136.

(58) Teranishi, T.; Hasegawa, S.; Shimizu, T.; Miyake, M. HeatInduced Size Evolution of Gold Nanoparticles in the Solid State. Adv. Mater. 2001, 13, 1699-1701.

(59) García-Morales, V.; Mafé, S. Monolayer-Protected Metallic Nanoparticles: Limitations of the Concentric Sphere Capacitor Model. J. Phys. Chem. C 2007, 111, 7242-7250.

(60) García-Morales, V.; Cervera, J.; Pellicer, J. Calculation of the Wetting Parameter from a Cluster Model in the Framework of Nanotermodynamics. Phys. Rev. E: Stat. Phys., Plasmas, Fluids, Relat. Interdiscip. Top. 2003, 67, 062103. 